\title{
@creative
}

\section{The number of edges of the edge polytope of a finite simple graph}

\author{
Takayuki Hibi \\ Department of Pure and Applied Mathematics, Graduate School of Information Science \\ and Technology, Osaka University, Toyonaka, Osaka 560-0043, Japan
}

\begin{abstract}
Aki Mori
Department of Pure and Applied Mathematics, Graduate School of Information Science and Technology, Osaka University, Toyonaka, Osaka 560-0043, Japan

Hidefumi Ohsugi

Department of Mathematical Sciences, School of Science and Technology, Kwansei Gakuin University, Sanda, Hyogo, 669-1337, Japan

Akihiro Shikama

Department of Pure and Applied Mathematics, Graduate School of Information Science and Technology, Osaka University, Toyonaka, Osaka 560-0043, Japan
\end{abstract}

Received 6 September 2014, accepted 8 October 2015, published online 5 February 2016

\begin{abstract}
Let $d \geq 3$ be an integer. It is known that the number of edges of the edge polytope of the complete graph with $d$ vertices is $d(d-1)(d-2) / 2$. In this paper, we study the maximum possible number $\mu_{d}$ of edges of the edge polytope arising from finite simple graphs with $d$ vertices. We show that $\mu_{d}=d(d-1)(d-2) / 2$ if and only if $3 \leq d \leq 14$. In addition, we study the asymptotic behavior of $\mu_{d}$. Tran-Ziegler gave a lower bound for $\mu_{d}$ by constructing a random graph. We succeeded in improving this bound by constructing both a non-random graph and a random graph whose complement is bipartite.
\end{abstract}

Keywords: Finite simple graph, edge polytope.

Math. Subj. Class.: 52B05, 05C30

E-mail addresses: hibi@math.sci.osaka-u.ac.jp (Takayuki Hibi), a-mori@cr.math.sci.osaka-u.ac.jp (Aki Mori), ohsugi@kwansei.ac.jp (Hidefumi Ohsugi), a-shikama@cr.math.sci.osaka-u.ac.jp (Akihiro Shikama)

(a) (i) This work is licensed under http://creativecommons.org/licenses/by/3.0/ 


\section{Introduction}

The number of $i$-dimensional faces of a convex polytope has been studied by many researchers for a long time. One of the most famous classical results is "Euler's formula." The extremal problem concerning the number of faces is an important topic in the study of convex polytopes. On the other hand, the study of edge polytopes of finite graphs has been conducted by many authors from viewpoints of commutative algebra on toric ideals and combinatorics of convex polytopes. We refer the reader to [2, 3] for foundations of edge polytopes. Faces of edge polytopes are studied in, e.g., [2, 4, 5]. Recently, Tran and Ziegler [6] studied this extremal problem on edge polytopes. In particular, using [5, Lemma 1.4], they gave bounds for the maximum possible number $\mu_{d}$ of edges of the edge polytope arising from finite simple graphs with $d$ vertices. Following [1, Question 1.3], we wish to find a finite simple graph $G$ with $d$ vertices such that the edge polytope of $G$ has $\mu_{d}$ edges and to compute $\mu_{d}$.

Recall that a finite simple graph is a finite graph with no loops and no multiple edges. Let $[d]=\{1, \ldots, d\}$ be the vertex set and $\Omega_{d}$ the set of finite simple graphs on $[d]$, where $d \geq 3$. Let $\mathbf{e}_{i}$ denote the $i$ th unit coordinate vector of the Euclidean space $\mathbb{R}^{d}$. Let $G \in \Omega_{d}$ and $E(G)$ the set of edges of $G$. If $e=\{i, j\} \in E(G)$, then we set $\rho(e)=\mathbf{e}_{i}+\mathbf{e}_{j} \in \mathbb{R}^{d}$. The edge polytope $\mathcal{P}_{G}$ of $G \in \Omega_{d}$ is the convex hull of the finite set $\{\rho(e): e \in E(G)\}$ in $\mathbb{R}^{d}$. Let $\varepsilon(G)$ denote the number of edges, namely 1-dimensional faces, of $\mathcal{P}_{G}$. For example, consider the case of the complete graph $K_{d}$ on $[d]$. By [5, Lemma 1.4], for edges $e$ and $f(e \neq f)$ of $K_{d}$, the convex hull of $\{\rho(e), \rho(f)\}$ is an edge of the edge polytope $\mathcal{P}_{K_{d}}$ if and only if $e$ and $f$ have a common vertex. Hence, $\varepsilon\left(K_{d}\right)=d\left(\begin{array}{c}d-1 \\ 2\end{array}\right)=d(d-1)(d-2) / 2$. On the other hand, $\varepsilon\left(K_{m, n}\right)=m n(m+n-2) / 2$, where $K_{m, n}$ is the complete bipartite graph on the vertex set $[m] \cup\{m+1, \ldots, m+n\}$ for which $m, n \geq 1$ (see [4, Theorem 2.5]). In this paper, we are interested in $\mu_{d}=\max \left\{\varepsilon(G): G \in \Omega_{d}\right\}$ for $d \geq 3$.

Theorem 1.1. For an integer $d \geq 3$, let $\Omega_{d}$ be the set of finite simple graphs on $[d]$. Given a graph $G \in \Omega_{d}$, let $\varepsilon(G)$ denote the number of edges of the edge polytope $\mathcal{P}_{G}$ of $G$. Then, the following holds:

(a) If $3 \leq d \leq 13$ and $G \in \Omega_{d}$ with $G \neq K_{d}$, then $\varepsilon(G)<\varepsilon\left(K_{d}\right)$.

(b) Let $G \in \Omega_{14}$ with $G \neq K_{14}$. Then $\varepsilon(G) \leq \varepsilon\left(K_{14}\right)$. Moreover, $\varepsilon(G)=\varepsilon\left(K_{14}\right)$ if and only if either $G=K_{14}-K_{4,5}$ or $G=K_{14}-K_{5,5}$.

(c) If $d \geq 15$, then there exists $G \in \Omega_{d}$ such that $\varepsilon(G)>\varepsilon\left(K_{d}\right)$.

We devote Section 2 to giving a proof of Theorem 1.1. At present, for $d \geq 15$, it remains unsolved to find $G \in \Omega_{d}$ with $\mu_{d}=\varepsilon(G)$ and to compute $\mu_{d}$. (Later, we will see that $\mu_{15} \geq \varepsilon\left(K_{15}\right)+50=1415$.) In Section 3 , we study the asymptotic behavior of $\mu_{d}$. Recently, Tran-Ziegler [6] gave a lower bound for $\mu_{d}$ by a random graph:

$$
\varepsilon(G(d, 1 / \sqrt{3}))=\frac{1}{54} d^{4}+\frac{1}{18} d^{3}-\frac{8}{27} d^{2}+\frac{1}{3} d .
$$

They also gave an upper bound for $\mu_{d}: \mu_{d} \leq\left(\frac{1}{32}+o(1)\right) d^{4}$. (However, this upper bound is not sharp. See [6, Remark].) In this paper, we succeeded in improving their lower bound by constructing a non-random graph (see Example 3.1) and a random graph whose complement is bipartite (see Theorem 3.2):

$$
\varepsilon(\mathbb{G})=\frac{5 \sqrt{5}-11}{8} d^{4}-\frac{12 \sqrt{5}-27}{2} d^{3}+\frac{19 \sqrt{5}-44}{2} d^{2}+d,
$$


where $\mathbb{G}=K_{d}-G\left(K_{d / 2, d / 2}, p\right)$ with $p=3-\sqrt{5}$. These results suggest the following:

Conjecture 1.2. Let $G \in \Omega_{d}$ with $\mu_{d}=\varepsilon(G)$. Then, the complement of $G$ is a bipartite graph.

Note that, by Theorem 1.1, this conjecture is true for $3 \leq d \leq 14$.

\section{Proof of Theorem 1.1}

In this section, we give a proof of Theorem 1.1. The following lemma is studied in [5, Lemma 1.4].

Lemma 2.1. Let $e$ and $f(e \neq f)$ be edges of a graph $G \in \Omega_{d}$. Then, the convex hull of $\{\rho(e), \rho(f)\}$ is an edge of the edge polytope $\mathcal{P}_{G}$ if and only if one of the following conditions is satisfied.

(i) e and $f$ have a common vertex in $[d]$.

(ii) $e=\{i, j\}$ and $f=\{k, l\}$ have no common vertices, and the induced subgraph of $G$ on the vertex set $\{i, j, k, l\}$ has no cycles of length 4 .

The complement graph $\bar{G}$ of a graph $G \in \Omega_{d}$ is the graph whose vertex set is $[d]$ and whose edges are the non-edges of $G$. For a vertex $i$ of a graph $G$, let $\operatorname{deg}_{G}(i)$ denote the degree of $i$ in $G$. We translate Lemma 2.1 in terms of the complement $\bar{G}$ of $G$.

Lemma 2.2. Let $H$ be the complement of a graph $G \in \Omega_{d}$. Then, we have

$$
\begin{aligned}
\varepsilon(G) & =\sum_{i=1}^{d}\left(\begin{array}{c}
d-1-\operatorname{deg}_{H}(i) \\
2
\end{array}\right)+a(H)+b(H)+c(H) \\
& =\varepsilon\left(K_{d}\right)+\frac{1}{2} \sum_{i=1}^{d} \operatorname{deg}_{H}^{2}(i)-(2 d-3)|E(H)|+a(H)+b(H)+c(H),
\end{aligned}
$$

where $a(H), b(H)$ and $c(H)$ are the number of induced subgraphs of $H$ on 4 vertices of the form (a) a path of length 3; (b) a cycle of length 4; (c) a path of length 2 and one isolated vertex, respectively.

Proof. First, the number of pairs of edges satisfying Lemma 2.1 (i) is equal to

$$
\begin{aligned}
\sum_{i=1}^{d}\left(\begin{array}{c}
d-1-\operatorname{deg}_{H}(i) \\
2
\end{array}\right) & =\sum_{i=1}^{d} \frac{(d-1)(d-2)-(2 d-3) \operatorname{deg}_{H}(i)+\operatorname{deg}_{H}^{2}(i)}{2} \\
& =\varepsilon\left(K_{d}\right)+\frac{1}{2} \sum_{i=1}^{d} \operatorname{deg}_{H}^{2}(i)-(2 d-3)|E(H)| .
\end{aligned}
$$

Second, the number of pairs of edges satisfying Lemma 2.1 (ii) is equal to the number of the induced subgraphs $W$ of $G$ where $W$ is one of the following: (a') $W$ is a path of length 3 ; (b') $W$ consists of two disjoint edges; (c') $W$ is a graph on $\{i, j, k, \ell\}$ with $E(W)=$ $\{\{i, j\},\{j, k\},\{i, k\},\{k, \ell\}\}$. Note that each induced subgraph has exactly one such pair of edges. The complement of each (a'), (b'), and (c') is (a), (b) and (c), respectively. 
For a graph $H \in \Omega_{r}$ with $r \leq d$, let $K_{d}-H$ denote the graph $G \in \Omega_{d}$ such that $E(G)=E\left(K_{d}\right) \backslash E(H)$. Using Lemma 2.2, we have the following:

Proposition 2.3. Let $H \in \Omega_{r}$ and let $\psi(H)$ denote the number of induced paths in $H$ of length 2. Then, the function $\varphi(d)=\varepsilon\left(K_{d}-H\right)-\varepsilon\left(K_{d}\right)$ for $d=r, r+1, r+2, \ldots$ is $a$ linear polynomial of $d$ whose leading coefficient is $\psi(H)-2|E(H)|$.

Proof. Since $d$ is a natural number it is sufficient to show that $\varphi(d+1)-\varphi(d)=\psi(H)-$ $2|E(H)|$ for any $d$. Let $H_{1}=\overline{K_{d}-H}$ and $H_{2}=\overline{K_{d+1}-H}$. Then, $H_{2}$ is obtained by adding one isolated vertex $d+1$ to $H_{1}$. Hence, it follows that $a\left(H_{1}\right)=a\left(H_{2}\right), b\left(H_{1}\right)=$ $b\left(H_{2}\right), c\left(H_{1}\right)+\psi(H)=c\left(H_{2}\right)$ and $\operatorname{deg}_{H_{1}}(i)=\operatorname{deg}_{H_{2}}(i)$ for all $1 \leq i \leq d$. Thus, by Lemma 2.2, we have

$$
\begin{aligned}
& \varphi(d+1)-\varphi(d) \\
= & \varepsilon\left(K_{d+1}-H\right)-\varepsilon\left(K_{d+1}\right)-\varepsilon\left(K_{d}-H\right)+\varepsilon\left(K_{d}\right) \\
= & \sum_{i=1}^{d+1}\left(\begin{array}{c}
d-\operatorname{deg}_{H_{2}}(i) \\
2
\end{array}\right)-\sum_{i=1}^{d}\left(\begin{array}{c}
d-1-\operatorname{deg}_{H_{1}}(i) \\
2
\end{array}\right)+\psi(H) \\
& +\frac{d(d-1)(d-2)}{2}-\frac{(d+1) d(d-1)}{2} \\
= & \left(\begin{array}{l}
d \\
2
\end{array}\right)+\sum_{i=1}^{d}\left(\left(\begin{array}{c}
d-\operatorname{deg}_{H_{1}}(i) \\
2
\end{array}\right)-\left(\begin{array}{c}
d-1-\operatorname{deg}_{H_{1}}(i) \\
2
\end{array}\right)\right)+\psi(H)-\frac{3 d(d-1)}{2} \\
= & \left(\begin{array}{l}
d \\
2
\end{array}\right)+\sum_{i=1}^{d}\left(d-1-\operatorname{deg}_{H_{1}}(i)\right)+\psi(H)-\frac{3 d(d-1)}{2} \\
= & \psi(H)-\sum_{i=1}^{d} \operatorname{deg}_{H_{1}}(i) \\
= & \psi(H)-2|E(H)|,
\end{aligned}
$$

as desired.

Proposition 2.4. Let $G \in \Omega_{d}$ and let $H_{1}, H_{2}, \ldots, H_{m}$ be all the nonempty connected components of $\bar{G}$. Then, $\varepsilon\left(K_{d}\right)-\varepsilon(G)=\sum_{j=1}^{m}\left(\varepsilon\left(K_{d}\right)-\varepsilon\left(K_{d}-H_{j}\right)\right)$.

Proof. Let $H=\bar{G}$ and let $H_{j}^{\prime}=\overline{K_{d}-H_{j}}$ for $1 \leq j \leq m$. Then, it is easy to see that $|E(H)|=\sum_{j=1}^{m}\left|E\left(H_{j}^{\prime}\right)\right|, \sum_{i=1}^{d} \operatorname{deg}_{H}^{2}(i)=\sum_{j=1}^{m} \sum_{i=1}^{d} \operatorname{deg}_{H_{j}^{\prime}}^{2}(i), a(H)=$ $\sum_{j=1}^{m} a\left(H_{j}^{\prime}\right), b(H)=\sum_{j=1}^{m} b\left(H_{j}^{\prime}\right)$, and $c(H)=\sum_{j=1}^{m} c\left(H_{j}^{\prime}\right)$. Thus, by Lemma 2.2, we are done.

A graph $G \in \Omega_{d}$ is called bipartite if $[d]$ admits a partition into two sets of vertices $V_{1}$ and $V_{2}$ such that, for every edge $\{i, j\}$ of $G$, either $i \in V_{1}, j \in V_{2}$ or $j \in V_{1}, i \in V_{2}$ is satisfied. A complete bipartite graph is a bipartite graph such that every pair of vertices $i, j$ with $i \in V_{1}$ and $j \in V_{2}$ is adjacent. Let $K_{m, n}$ denote the complete bipartite graph with $\left|V_{1}\right|=m$ and $\left|V_{2}\right|=n$.

Proposition 2.5. Let $G=K_{d}-K_{m, n}$ such that $m+n \leq d$ and $m, n \geq 1$. Then,

$$
\varepsilon(G)-\varepsilon\left(K_{d}\right)=\frac{1}{2} m n(m+n-6) d-\frac{1}{4} m n\left(3 m n+2 m^{2}+2 n^{2}-5 m-5 n-13\right) .
$$


Proof. Let $H=K_{m, n}$. Then,

$$
\psi(H)-2|E(H)|=m\left(\begin{array}{l}
n \\
2
\end{array}\right)+n\left(\begin{array}{c}
m \\
2
\end{array}\right)-2 m n=\frac{1}{2} m n(m+n-6) .
$$

Moreover, since $K_{m+n}-K_{m, n}$ is the disjoint union of $K_{m}$ and $K_{n}$, we have

$$
\begin{aligned}
\varphi(m+n)= & \frac{m(m-1)(m-2)}{2}+\frac{n(n-1)(n-2)}{2}+\left(\begin{array}{c}
m \\
2
\end{array}\right)\left(\begin{array}{l}
n \\
2
\end{array}\right) \\
& -\frac{(m+n)(m+n-1)(m+n-2)}{2} \\
= & \frac{1}{4} m n(m n-7 m-7 n+13)
\end{aligned}
$$

by Lemma 2.1. Hence, by Proposition 2.3,

$$
\begin{aligned}
\varepsilon(G)-\varepsilon\left(K_{d}\right) & =\frac{1}{2} m n(m+n-6)(d-(m+n))+\frac{1}{4} m n(m n-7 m-7 n+13) \\
& =\frac{1}{2} m n(m+n-6) d-\frac{1}{4} m n\left(3 m n+2 m^{2}+2 n^{2}-5 m-5 n-13\right),
\end{aligned}
$$

as desired.

Let $k_{3}(H)$ denote the number of triangles (i.e., cycles of length 3 ) of $H$. The following lemma is important.

Lemma 2.6. Let $H$ be the complement graph of $G \in \Omega_{d}$. Then, we have

$$
\varepsilon(G) \leq \varepsilon\left(K_{d}\right)+\frac{d^{2}-16 d+29}{7}|E(H)|-\frac{3}{7}(d-8) k_{3}(H) .
$$

Proof. The number of pairs of edges satisfying Lemma 2.1 (i) is, by Lemma 2.2, $\varepsilon\left(K_{d}\right)-$ $(2 d-3)|E(H)|+\frac{1}{2} \sum_{i=1}^{d} \operatorname{deg}_{H}^{2}(i)$. For an edge $\{i, j\}$ of $H$, let $k_{3}(i, j)$ be the number of triangles in $H$ containing $\{i, j\}$. We define three subsets of $[d] \backslash\{i, j\}$ :

$$
\begin{aligned}
X_{i, j} & =\{\ell \in[d] \backslash\{i, j\}:\{i, \ell\} \in E(H),\{j, \ell\} \notin E(H)\}, \\
Y_{i, j} & =\{\ell \in[d] \backslash\{i, j\}:\{j, \ell\} \in E(H),\{i, \ell\} \notin E(H)\}, \\
Z_{i, j} & =\{\ell \in[d] \backslash\{i, j\}:\{i, \ell\} \notin E(H),\{j, \ell\} \notin E(H)\} .
\end{aligned}
$$

It then follows that, $\left|X_{i, j}\right|+\left|Y_{i, j}\right|+\left|Z_{i, j}\right|+k_{3}(i, j)=d-2$, and

$$
\begin{aligned}
\frac{1}{2} \sum_{i=1}^{d} \operatorname{deg}_{H}^{2}(i) & =\frac{1}{2} \sum_{\{i, j\} \in E(H)}\left(\operatorname{deg}_{H}(i)+\operatorname{deg}_{H}(j)\right) \\
& =\frac{1}{2} \sum_{\{i, j\} \in E(H)}\left(\left|X_{i, j}\right|+\left|Y_{i, j}\right|+2 k_{3}(i, j)+2\right) \\
& =|E(H)|+3 k_{3}(H)+\frac{1}{2} \sum_{\{i, j\} \in E(H)}\left(\left|X_{i, j}\right|+\left|Y_{i, j}\right|\right)
\end{aligned}
$$


Second, we count the number of pairs satisfying Lemma 2.1 (ii). By Lemma 2.2, this number is equal to $a(H)+b(H)+c(H)$. Here, we count the number of the induced subgraphs $H^{\prime}$ of type (a), (b) and (c) containing an edge $e=\{i, j\}$ of $H$. If $e$ is an edge of $H^{\prime}$, then the other two vertices $\ell$ and $m$ of $H^{\prime}$ satisfy exactly one of the following conditions:

(i) $\ell \in X_{i, j}, m \in Y_{i, j}$;

(ii) $\ell \in Y_{i, j}, m \in Z_{i, j}$;

(iii) $\ell \in Z_{i, j}, m \in X_{i, j}$.

If $i, j, \ell, m$ satisfy condition (i), then one of the following holds:

- $H^{\prime}$ is a path $\left(e_{1}, e_{2}, e_{3}\right)$ and $e=e_{2}$ (type (a));

- $H^{\prime}$ is a cycle of length 4 and $e$ is one of four edges (type (b)).

It then follows that

$$
a(H)+4 b(H)=\sum_{\{i, j\} \in E(H)}\left|X_{i, j}\right|\left|Y_{i, j}\right| .
$$

If $i, j, \ell, m$ satisfy either condition (ii) or (iii), then one of the following holds:

- $H^{\prime}$ is a path $\left(e_{1}, e_{2}, e_{3}\right)$ and $e \in\left\{e_{1}, e_{3}\right\}$ (type (a));

- $H^{\prime}$ is a path $\left(e_{1}, e_{2}\right)$ with one isolated vertex and $e \in\left\{e_{1}, e_{2}\right\}$ (type (c)).

It then follows that

$$
2 a(H)+2 c(H)=\sum_{\{i, j\} \in E(H)}\left(\left|Y_{i, j}\right|\left|Z_{i, j}\right|+\left|Z_{i, j}\right|\left|X_{i, j}\right|\right) .
$$

Thus, we have

$$
a(H)+b(H)+c(H)=-\frac{a(H)}{4}+\sum_{\{i, j\} \in E(H)}\left(\frac{1}{4}\left|X_{i, j}\right|\left|Y_{i, j}\right|+\frac{1}{2}\left|Y_{i, j}\right|\left|Z_{i, j}\right|+\frac{1}{2}\left|Z_{i, j}\right|\left|X_{i, j}\right|\right) .
$$

Subject to $\left|X_{i, j}\right|+\left|Y_{i, j}\right|+\left|Z_{i, j}\right|=d-2-k_{3}(i, j)$, we study an upper bound of

$$
\alpha=\sum_{\{i, j\} \in E(H)}\left(\frac{\left|X_{i, j}\right|+\left|Y_{i, j}\right|}{2}+\frac{1}{4}\left|X_{i, j}\right|\left|Y_{i, j}\right|+\frac{1}{2}\left|Y_{i, j}\right|\left|Z_{i, j}\right|+\frac{1}{2}\left|Z_{i, j}\right|\left|X_{i, j}\right|\right) .
$$

Each summand of $\alpha$ satisfies

$$
\begin{aligned}
& \frac{\left|X_{i, j}\right|+\left|Y_{i, j}\right|}{2}+\frac{1}{4}\left|X_{i, j}\right|\left|Y_{i, j}\right|+\frac{1}{2}\left|Y_{i, j}\right|\left|Z_{i, j}\right|+\frac{1}{2}\left|Z_{i, j}\right|\left|X_{i, j}\right| \\
= & \frac{1}{4}\left|X_{i, j}\right|\left|Y_{i, j}\right|+\frac{1}{2}\left(\left|X_{i, j}\right|+\left|Y_{i, j}\right|\right)\left(d-1-k_{3}(i, j)-\left(\left|X_{i, j}\right|+\left|Y_{i, j}\right|\right)\right) \\
\leq & \frac{1}{4}\left(\frac{\left|X_{i, j}\right|+\left|Y_{i, j}\right|}{2}\right)^{2}+\frac{1}{2}\left(\left|X_{i, j}\right|+\left|Y_{i, j}\right|\right)\left(d-1-k_{3}(i, j)-\left(\left|X_{i, j}\right|+\left|Y_{i, j}\right|\right)\right) \\
= & -\frac{7}{16}\left(\left|X_{i, j}\right|+\left|Y_{i, j}\right|\right)^{2}+\frac{d-1-k_{3}(i, j)}{2}\left(\left|X_{i, j}\right|+\left|Y_{i, j}\right|\right) .
\end{aligned}
$$


The last function has the maximum number $\frac{1}{7}\left(d-1-k_{3}(i, j)\right)^{2}$ when $\left|X_{i, j}\right|+\left|Y_{i, j}\right|=$ $\frac{4}{7}\left(d-1-k_{3}(i, j)\right)$. Hence,

$$
\begin{aligned}
\sum_{\{i, j\} \in E(H)} \frac{1}{7}\left(d-1-k_{3}(i, j)\right)^{2} & \leq \sum_{\{i, j\} \in E(H)} \frac{1}{7}(d-1)\left(d-1-k_{3}(i, j)\right) \\
& =\frac{1}{7} \sum_{\{i, j\} \in E(H)}(d-1)^{2}-\frac{1}{7} \sum_{\{i, j\} \in E(H)}(d-1) k_{3}(i, j) \\
& =\frac{1}{7}(d-1)^{2}|E(H)|-\frac{3}{7}(d-1) k_{3}(H)
\end{aligned}
$$

is an upper bound of $\alpha$. Thus,

$$
\varepsilon\left(K_{d}\right)-(2 d-3)|E(H)|+|E(H)|+3 k_{3}(H)+\frac{1}{7}(d-1)^{2}|E(H)|-\frac{3}{7}(d-1) k_{3}(H)
$$

is an upper bound of $\varepsilon(G)$ as desired.

Using Proposition 2.5 and Lemma 2.6, we prove Theorem 1.1.

Proof of Theorem 1.1. (a) Let $3 \leq d \leq 13$ and $G \in \Omega_{d}$ with $G \neq K_{d}$. If $d=3$, then $\varepsilon(G)<\varepsilon\left(K_{d}\right)$ is trivial. If $d=4$, then $\varepsilon\left(K_{4}\right)=12$. Since $|E(G)|<6$, we have $\varepsilon(G) \leq\left(\begin{array}{l}5 \\ 2\end{array}\right)=10<\varepsilon\left(K_{4}\right)$. Let $d \geq 5$ and let $H$ be the complement graph of $G$. By Lemma 2.6,

$$
\varepsilon(G)-\varepsilon\left(K_{d}\right) \leq \frac{d^{2}-16 d+29}{7}|E(H)|-\frac{3}{7}(d-8) k_{3}(H) .
$$

If $8 \leq d \leq 13$, then $\varepsilon(G)-\varepsilon\left(K_{d}\right)<0$ since $\frac{d^{2}-16 d+29}{7}<0,|E(H)|>0$ and $k_{3}(H) \geq 0$. Let $5 \leq d \leq 7$. Then,

$$
\varepsilon(G)-\varepsilon\left(K_{d}\right) \leq \begin{cases}-\frac{26}{7}|E(H)|+\frac{9}{7} k_{3}(H) & \text { if } d=5, \\ -\frac{31}{7}|E(H)|+\frac{6}{7} k_{3}(H) & \text { if } d=6, \\ -\frac{34}{7}|E(H)|+\frac{3}{7} k_{3}(H) & \text { if } d=7 .\end{cases}
$$

Hence, if $k_{3}(H) \leq 2$, then $\varepsilon(G)-\varepsilon\left(K_{d}\right)$ is negative. On the other hand, if $k_{3}(H) \geq 3$, then $|E(H)| \geq 5$. Since $k_{3}(H) \leq\left(\begin{array}{l}d \\ 3\end{array}\right)$, it follows that $\varepsilon(G)-\varepsilon\left(K_{d}\right)$ is negative.

(b) Let $G \in \Omega_{14}$ with $G \neq K_{14}$ and let $H=\bar{G}$. We need to evaluate the function which appears in the proof of Lemma 2.6 more accurately by focusing on $d=14$. Let $\left|Z_{i, j}\right|=12-k_{3}(i, j)-\left|X_{i, j}\right|-\left|Y_{i, j}\right|$ and

$$
\begin{aligned}
f & =\frac{\left|X_{i, j}\right|+\left|Y_{i, j}\right|}{2}+\frac{1}{4}\left|X_{i, j}\right|\left|Y_{i, j}\right|+\frac{1}{2}\left|Y_{i, j}\right|\left|Z_{i, j}\right|+\frac{1}{2}\left|Z_{i, j}\right|\left|X_{i, j}\right| \\
g & =-\frac{7}{16}\left(\left|X_{i, j}\right|+\left|Y_{i, j}\right|\right)^{2}+\frac{13-k_{3}(i, j)}{2}\left(\left|X_{i, j}\right|+\left|Y_{i, j}\right|\right)
\end{aligned}
$$

be functions of $\left|X_{i, j}\right|$ and $\left|Y_{i, j}\right|$. Recall that $f \leq g \leq \frac{1}{7}\left(13-k_{3}(i, j)\right)^{2}$ and $g=\frac{1}{7}(13-$ $\left.k_{3}(i, j)\right)^{2}$ when $\left|X_{i, j}\right|+\left|Y_{i, j}\right|=\frac{4}{7}\left(13-k_{3}(i, j)\right)$. If $1 \leq k_{3}(i, j) \leq 12$, then

$\frac{1}{7}\left(13-k_{3}(i, j)\right)^{2}=24-\frac{13}{7} k_{3}(i, j)-\frac{11}{7}+\frac{1}{7}\left(k_{3}(i, j)-1\right)\left(k_{3}(i, j)-12\right)<24-\frac{13}{7} k_{3}(i, j)$. 
If $k_{3}(i, j)=0$, then $\frac{1}{7}\left(13-k_{3}(i, j)\right)^{2}=24+1 / 7$. However, since

$$
4\left(\frac{\left|X_{i, j}\right|+\left|Y_{i, j}\right|}{2}+\frac{1}{4}\left|X_{i, j}\right|\left|Y_{i, j}\right|+\frac{1}{2}\left|Y_{i, j}\right|\left|Z_{i, j}\right|+\frac{1}{2}\left|Z_{i, j}\right|\left|X_{i, j}\right|\right)
$$

is an integer, the value of $f$ is at most 24 if $\left|X_{i, j}\right|$ and $\left|Y_{i, j}\right|$ are non-negative integers. Thus, for $k_{3}(i, j)=0,1, \ldots, 12$, the value of $f$ is at most $24-\frac{13}{7} k_{3}(i, j)$ if $\left|X_{i, j}\right|$ and $\left|Y_{i, j}\right|$ are non-negative integers. Thus, by the same argument in the proof of Lemma 2.6, $\varepsilon(G)-\varepsilon\left(K_{14}\right)$ is at most

$-24|E(H)|+3 k_{3}(H)+24|E(H)|-\frac{3 \cdot 13}{7} k_{3}(H)-\frac{a(H)}{4}=-\frac{18}{7} k_{3}(H)-\frac{a(H)}{4} \leq 0$.

Therefore, $\varepsilon(G) \leq \varepsilon\left(K_{14}\right)$.

Suppose that $\varepsilon(G)=\varepsilon\left(K_{14}\right)$. Then, $-\frac{18}{7} k_{3}(H)-\frac{a(H)}{4} \geq 0$. Since $k_{3}(H), a(H) \geq 0$, we have $k_{3}(H)=a(H)=0$. Moreover,

$$
\frac{\left|X_{i, j}\right|+\left|Y_{i, j}\right|}{2}+\frac{1}{4}\left|X_{i, j}\right|\left|Y_{i, j}\right|+\frac{1}{2}\left|Y_{i, j}\right|\left|Z_{i, j}\right|+\frac{1}{2}\left|Z_{i, j}\right|\left|X_{i, j}\right|=24
$$

and $\left|X_{i, j}\right|+\left|Y_{i, j}\right|+\left|Z_{i, j}\right|=12$ for an arbitrary edge $\{i, j\}$ of $H$. It is easy to see that $\left|X_{i, j}\right|+\left|Y_{i, j}\right| \in\{7,8\}$. It then follows that, for an arbitrary $\{i, j\} \in E(H),\left(\left|X_{i, j}\right|,\left|Y_{i, j}\right|\right.$, $\left.\left|Z_{i, j}\right|\right) \in\{(3,4,5),(4,3,5),(4,4,4)\}$. In particular, the degree of each vertex is either 0 , 4 or 5 . Moreover, since $k_{3}(H)=0,\{j\} \cup X_{i, j}$ and $\{i\} \cup Y_{i, j}$ are independent sets. Hence, by $a(H)=0$, the induced subgraph of $H$ on $\{i, j\} \cup X_{i, j} \cup Y_{i, j}$ is the complete bipartite graph $K_{\left|X_{i, j}\right|+1,\left|Y_{i, j}\right|+1}$.

Suppose that an edge $\{i, j\}$ of $H$ satisfies $\left(\left|X_{i, j}\right|,\left|Y_{i, j}\right|,\left|Z_{i, j}\right|\right)=(4,4,4)$. Then, the induced subgraph of $H$ on $\{i, j\} \cup X_{i, j} \cup Y_{i, j}$ is $K_{5,5}$. Since the degree of any vertex of $H$ is either, 0,4 or 5 , other four vertices are isolated. Therefore, $G=K_{14}-K_{5,5}$.

It is enough to consider the case that $\left(\left|X_{s, t}\right|,\left|Y_{s, t}\right|,\left|Z_{s, t}\right|\right) \neq(4,4,4)$ holds for every edge $\{s, t\}$. Suppose that $\left(\left|X_{i, j}\right|,\left|Y_{i, j}\right|\right)=(3,4)$. Then, the induced subgraph of $H$ on $\{i, j\} \cup X_{i, j} \cup Y_{i, j}$ is $K_{4,5}$. Since $\left(\left|X_{s, t}\right|,\left|Y_{s, t}\right|,\left|Z_{s, t}\right|\right) \neq(4,4,4)$ for each edge $\{s, t\}$, the degree of every vertex in $\{i\} \cup Y_{i, j}$ is 4 . In this case, $K_{4,5}$ is a connected component of $H$. Since the degree of other five vertices is at most 4 , it follows that they are isolated vertices. Therefore, $G=K_{14}-K_{4,5}$.

(c) Let $d \geq 15$ and let $G=K_{d}-K_{m, n} \in \Omega_{d}$. By Proposition 2.5, we have

$$
\varepsilon(G)-\varepsilon\left(K_{d}\right)=\frac{1}{2} m n(m+n-6) d-\frac{1}{4} m n\left(3 m n+2 m^{2}+2 n^{2}-5 m-5 n-13\right) .
$$

When $m=n=5$, we obtain $\varepsilon(G)-\varepsilon\left(K_{d}\right)=50(d-14)>0$ as desired.

\section{Asymptotic behavior of $\mu_{d}$}

For $0<p<1$ and an integer $d>0$, let $G(d, p)$ denote the random graph on the vertex set $[d]$ in which the edges are chosen independently with probability $p$. For a graph $H$ on the vertex set $[d]$ and $0<p<1$, let $G(H, p)$ denote the random graph on the vertex set $[d]$ in which the edges of $H$ are chosen independently with probability $p$ and the edges not belonging to $H$ are not chosen. Tran-Ziegler [6] showed that, for the random graph $G(d, 1 / \sqrt{3})$,

$$
\varepsilon(G(d, 1 / \sqrt{3}))=\frac{1}{54} d^{4}+\frac{1}{18} d^{3}-\frac{8}{27} d^{2}+\frac{1}{3} d,
$$


and hence this is a lower bound for $\mu_{d}$.

First, for $d \gg 0$, we give an example of a (non-random) graph $G$ on the vertex set $[d]$ such that $\varepsilon(G)>\varepsilon(G(d, 1 / \sqrt{3}))$.

Example 3.1. Let $G=K_{d}-K_{a d, a d}-K_{(1 / 2-a) d,(1 / 2-a) d}$ where $a=\frac{1}{28}(7+\sqrt{21})$ and $d \gg 0$. By Propositions 2.4 and 2.5, it follows that

$$
\varepsilon(G)=\frac{9}{448} d^{4}+\frac{1}{7} d^{3}-\frac{103}{112} d^{2}+d .
$$

Since $1 / 54 \fallingdotseq 0.0185$ and $9 / 448 \fallingdotseq 0.0201$, we have $\varepsilon(G)>\varepsilon(G(d, 1 / \sqrt{3}))$ for $d \gg 0$.

Second, we give a random graph $\mathbb{G}$ on the vertex set $[d]$ such that $\varepsilon(\mathbb{G})>\varepsilon(G(d, 1 / \sqrt{3}))$ for $d \gg 0$.

Theorem 3.2. For an integer $d$, let $\mathbb{G}$ be a random graph $K_{d}-G\left(K_{d / 2, d / 2}, p\right)$ with $p=$ $3-\sqrt{5}$. Then,

$$
\varepsilon(\mathbb{G})=\frac{5 \sqrt{5}-11}{8} d^{4}-\frac{12 \sqrt{5}-27}{2} d^{3}+\frac{19 \sqrt{5}-44}{2} d^{2}+d .
$$

In particular, we have $\varepsilon(\mathbb{G})>\varepsilon(G(d, 1 / \sqrt{3}))$ for all $d \gg 0$.

Proof. Let $m=d / 2$ and let $[d]=V_{1} \cup V_{2}$ be a partition of the vertex set of $K_{m, m}$. The number of pairs of edges $\{i, j\},\{i, k\}$ satisfying Lemma 2.1 (i) is

$$
\eta_{1}=m(m-1)(m-2)+2 m^{2}(m-1)(1-p)+m^{2}(m-1)(1-p)^{2}
$$

where each term corresponds to the case when (i) $i, j, k \in V_{s}$, (ii) $i, j \in V_{s}, k \notin V_{s}$ and (iii) $i \in V_{s}, j, k \notin V_{s}$, respectively.

Next, we study the number of pairs of edges $\{i, j\},\{k, \ell\}$ satisfying Lemma 2.1 (ii). Let $\mathbb{G}_{i j k \ell}$ denote the induced subgraph of $\mathbb{G}$ on the vertex set $\{i, j, k, \ell\} \subset[d]$. If either " $i, j, k, \ell \in V_{s}$ " or " $i, \ell \in V_{s}$ and $j, k \notin V_{s}$ " holds, then $\{i, j, k, \ell\}$ is a cycle of $\mathbb{G}_{i j k \ell}$ whenever $\{i, j\},\{k, \ell\} \in E(\mathbb{G})$. Hence, we consider the following two cases:

Case 1. Suppose $i, j \in V_{s}$ and $k, \ell \notin V_{s}$. Then, $\mathbb{G}_{i j k \ell}$ has a cycle of length 4 if and only if either $\{i, k\},\{j, \ell\} \in E(\mathbb{G})$ or $\{i, \ell\},\{j, k\} \in E(\mathbb{G})$ holds. Thus, the expected number of pairs of edges is $\eta_{2}=\left(\begin{array}{c}m \\ 2\end{array}\right)^{2}\left(1-(1-p)^{2}\right)^{2}$.

Case 2. Suppose that $i \in V_{s}$ and $j, k, \ell \notin V_{s}$ hold. Then, all of $\{k, \ell\},\{j, k\}$ and $\{j, \ell\}$ are edges of $\mathbb{G}$. On the other hand, $\{i, j\}$ is an edge of $\mathbb{G}$ with probability $1-p$. If $\{i, j\}$ is an edge of $\mathbb{G}$, then $\mathbb{G}_{i j k \ell}$ has a cycle of length 4 if and only if either $\{i, k\} \in E(\mathbb{G})$ or $\{i, \ell\} \in E(\mathbb{G})$ holds. Thus, the expected number of pairs of edges is $\eta_{3}=m^{2}(m-1)(m-2)(1-p) p^{2}$.

Therefore, $\varepsilon(\mathbb{G})=\eta_{1}+\eta_{2}+\eta_{3}$. If $m=d / 2$ and $p=3-\sqrt{5}$, then

$$
\varepsilon(\mathbb{G})=\frac{5 \sqrt{5}-11}{8} d^{4}-\frac{12 \sqrt{5}-27}{2} d^{3}+\frac{19 \sqrt{5}-44}{2} d^{2}+d,
$$

whose leading coefficient is $\frac{5 \sqrt{5}-11}{8} \fallingdotseq 0.0225425$. 
Remark 3.3. By Theorem 3.2, the graph $G$ in Example 3.1 does not satisfy $\mu_{d}=\varepsilon(G)$ for $d \gg 0$. In fact, for $d=20$, by Propositions 2.4 and 2.5, it follows that

$$
\max \left\{\varepsilon(G): \quad \begin{array}{c}
G \in \Omega_{20} \text { and each non-empty connected } \\
\text { component of } \bar{G} \text { is a complete bipartite graph }
\end{array}\right\}=4176 .
$$

Let $G^{\prime} \in \Omega_{20}$ be the graph such that $\overline{G^{\prime}}$ is the bipartite graph with $E\left(\overline{G^{\prime}}\right)=$

$$
\begin{gathered}
\{\{1,12\},\{1,14\},\{1,15\},\{1,16\},\{1,18\},\{1,19\},\{1,20\},\{2,11\},\{2,12\},\{2,13\},\{2,15\}, \\
\{2,17\},\{2,19\},\{2,20\},\{3,11\},\{3,12\},\{3,13\},\{3,14\},\{3,15\},\{3,16\},\{3,18\},\{4,14\} \\
\{4,15\},\{4,16\},\{4,17\},\{4,18\},\{4,19\},\{4,20\},\{5,11\},\{5,12\},\{5,13\},\{5,15\},\{5,17\} \\
\{5,18\},\{5,20\},\{6,12\},\{6,16\},\{6,17\},\{6,18\},\{6,19\},\{6,20\},\{7,11\},\{7,12\},\{7,13\}, \\
\{7,14\},\{7,16\},\{7,17\},\{7,19\},\{8,11\},\{8,12\},\{8,13\},\{8,14\},\{8,15\},\{8,18\},\{8,19\} \\
\{8,20\},\{9,11\},\{9,14\},\{9,15\},\{9,16\},\{9,17\},\{9,18\},\{9,19\},\{10,11\},\{10,13\},\{10,15\}, \\
\{10,16\},\{10,18\},\{10,19\},\{10,20\}\} .
\end{gathered}
$$

Then, $\varepsilon\left(G^{\prime}\right)=4203>4176$.

Acknowledgment. The authors are grateful to an anonymous referee for useful suggestions, and helpful comments.

\section{References}

[1] T. Hibi, N. Li and Y. X. Zhang, Separating hyperplanes of edge polytopes, J. Combin. Theory Ser. A 120 (2013), 218-231, doi:10.1016/j.jcta.2012.08.002.

[2] H. Ohsugi and T. Hibi, Normal polytopes arising from finite graphs, J. Algebra 207 (1998), 409-426, doi:10.1006/jabr.1998.7476.

[3] H. Ohsugi and T. Hibi, Toric ideals generated by quadratic binomials, J. Algebra 218 (1999), 509-527, doi:10.1006/jabr.1999.7918.

[4] H. Ohsugi and T. Hibi, Compressed polytopes, initial ideals and complete multipartite graphs, Illinois J. Math. 44 (2000), 391-406.

[5] H. Ohsugi and T. Hibi, Simple polytopes arising from finite graphs, in: Proceedings of the 2008 International Conference on Information Theory and Statistical Learning (ITSL), 2008 pp. 7379, (available at http: / /arxiv.org/abs/0804.4287).

[6] T. Tran and G. M. Ziegler, Extremal edge polytopes, Electron. J. Combin. 21 (2014), Paper 2.57, 16. 